\title{
Personalien
}

Praxiseröffnungen /

Nouveaux cabinets médicaux /

Nuovi studi medici

GE

\section{Aline Richard,}

Spécialiste en pédiatrie, Clinique de Carouge, Avenue Cardinal Mermillod 1,

1227 Carouge GE

\section{SO}

\section{Giacomo Duchini,}

Facharzt für Dermatologie und Venerologie und Facharzt für Allgemeine Innere Medizin, Praxis Dermatologie Aare, Westbahnhofstrasse 1, 4500 Solothurn
Ärztegesellschaft des Kantons Bern

Ärztlicher Bezirksverein Bern Regio Zur Aufnahme als ordentliches Mitglied in leitender Tätigkeit hat sich angemeldet:

Roman Trepp, Facharzt für Allgemeine Innere Medizin und Facharzt für Endokrinologie/ Diabetologie, FMH, Leitender Arzt Endokrinologie, UDEM Inselspital, 3010 Bern

Einsprachen gegen dieses Vorhaben müssen innerhalb 14 Tagen seit der Veröffentlichung schriftlich und begründet bei den Co-Präsidenten des Ärztlichen Bezirksvereins Bern Regio eingereicht werden. Nach Ablauf der Frist entscheidet der Vorstand über die Aufnahme der Gesuche und über die allfälligen Einsprachen.

\section{Ärztegesellschaft Thurgau \\ Zum Eintritt in die Ärztegesellschaft Thurgau hat sich gemeldet:}

Peter Rohr, Facharzt für Gastroenterologie, FMH, Torggelstrasse 12, 8265 Mammern

Ärzte-Gesellschaft des Kantons Zug

Zur Aufnahme in die Ärzte-Gesellschaft des Kantons Zug als ordentliches Mitglied hat sich angemeldet:

Andreas Burkhart, Facharzt für Allgemeine Innere Medizin, FMH, Poststrasse 22, $6300 \mathrm{Zug}$

Einsprachen gegen diese Kandidatur müssen innerhalb 14 Tagen seit dieser Veröffentlichung schriftlich und begründet beim Sekretariat der Ärzte-Gesellschaft des Kantons Zug eingereicht werden. Nach Ablauf der Einsprachefrist entscheidet der Vorstand über Gesuch und allfällige Einsprachen.

\footnotetext{
Aktuelle Themen auf unserer Website www.saez.ch $\rightarrow$ tour d'horizon

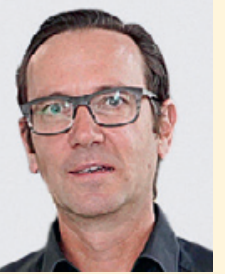
Interview mit Christian Eissler, Studiengangsleiter «MSc Pflege», Berner Fachhochschule «Ein wichtiger Bestandteil gelebter Integrierter Versorgung»

Die Integration neuer Berufsgruppen wie Nurse Practitioner als zentraler Lösungsansatz, um dem Fachkräftemangel in der Grundversorgung und Pflege zu begegnen.

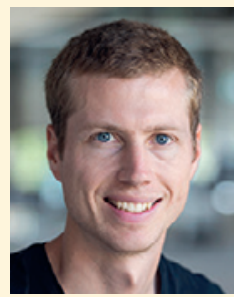

Interview mit Roland Sigrist, Executive Director \& Head of Finances Cybathlon

\section{«Es gibt keinen vergleichbaren Wettkampf»}

Am Cybathlon messen sich Menschen mit körperlicher Beeinträchtigung mithilfe modernster Technologien. Der Wettkampf soll Innovationen und die Forschung in diesem Bereich fördern.
} 